\title{
Special relativistic magnetohydrodynamic simulation of two-component outflow powered by magnetic explosion on compact stars
}

\author{
Jin Matsumoto ${ }^{1,2}$, Youhei Masada ${ }^{3,4}$, \\ ${\text { Eiji } \text { Asano }^{1} \text { and Kazunari Shibata }}^{1}$ \\ ${ }^{1}$ Kwasan and Hida Observatories, Kyoto University, Kyoto, Japan \\ email: jin@kusastro.kyoto-u.ac.jp \\ ${ }^{2}$ Department of Astronomy, Kyoto University, Kyoto, Japan \\ ${ }^{3}$ Graduate School of System Informatics, Department of Computational Science, \\ Kobe University, Kobe, Japan \\ ${ }^{4}$ Hinode Science Project, National Astronomical Observatory of Japan, Tokyo, Japan
}

\begin{abstract}
The nonlinear dynamics of the outflow driven by magnetic explosion on the surface of compact object is investigated through special relativistic magnetohydrodynamic simulations. We adopt, as an initial equilibrium state, a spherical stellar object embedded in the hydrostatic plasma which has a density $\rho(r) \propto r^{-\alpha}$ and is threaded by a dipole magnetic field. The injection of magnetic energy at the surface of compact star breaks the dynamical equilibrium and triggers two-component outflow. At the early evolutionary stage, the magnetic pressure increases rapidly in time around the stellar surface, initiating a magnetically driven outflow. Then it excites a strong forward shock, shock driven outflow. The expansion velocity of the magnetically driven outflow is characterized by the Alfvén velocity on the stellar surface, and follows a simple scaling relation $v_{\mathrm{mag}} \propto v_{\mathrm{A}}{ }^{1 / 2}$. When the initial density profile declines steeply with radius, the strong shock is accelerated self-similarly to relativistic velocity ahead of the magnetically driven component. We find that the evolution of the strong forward shock can be described by a self-similar relation $\Gamma_{\mathrm{sh}} \propto r_{\mathrm{sh}}$, where $\Gamma_{\mathrm{sh}}$ is the Lorentz factor of the plasma measured at the shock surface $r_{\mathrm{sh}}$. It should be stressed that the pure hydrodynamic process is responsible for the acceleration of the shock driven outflow. Our two-component outflow model, which is the natural outcome of the magnetic explosion, would deepen the understanding of the magnetic active phenomena on various magnetized stellar objects.
\end{abstract}

Keywords. relativistic, MHD, neutron stars, numerical

\section{Introduction}

Plasma outflow from gravitationally bounded stellar objects is a universal phenomenon in astrophysics although it has different spatial and energetic scales. The energetic origin of plasma outflows would be different in the various astrophysical systems. However, there should be a characteristic common to all the outflow phenomena. The magnetohydrodynamic processes play essential roles in powering and regulating the outflow from a lot of stelar and black-hole systems. It has been a central issue in the outflow study to identify the MHD process which controls the outflow dynamics (Hayashi et al. 1996; Beskin \& Nokhrina 2006; Spruit 2010).

In this context, the physical properties and the acceleration mechanism of the plasma outflow powered by the magnetic energy stored in compact stars have not been studied sufficiently yet although they are important not only in understanding active phenomena 


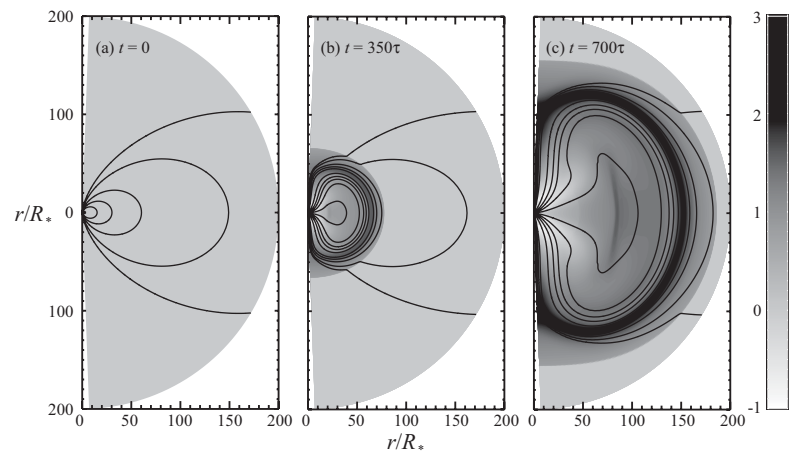

Figure 1. The time evolution of the density distribution (log scale) and magnetic field lines in the meridional plane for the case where $\alpha=3.50$ and $\beta_{0}=3.2$. The density is normalized by its initial value: $\rho(r, \theta, t) / \rho(r, \theta, 0)$. The left, middle and right panels are corresponding to those in $t / \tau=0,350$, and 700 respectively. The normalization unit in time is the light crossing time $\tau \equiv R_{*} / c$ where $R_{*}$ and $c$ are central star radius and light speed, respectively.

on the neutron star/magnetar (Thompson \& Duncan 1995), but also in unifying the driving mechanisms of the astrophysical outflow. It is interesting from a physical point of view that the outflow from such compact object can be accelerated to the relativistic velocity (Komissarov \& Lyubarsky 2004; Komissarov 2006; Takahashi et al. 2009).

We focus on the plasma outflows powered by a magnetic explosions on a dense compact object. We investigate their nonlinear dynamics using axisymmetric special relativistic MHD simulations. As an initial setting, we set a spherical compact object embedded in the hydrostatic gaseous plasma which has a density $\rho(r) \propto r^{-\alpha}$ and is threaded by a dipole magnetic field where $r$ and $\alpha$ are the distance from the centroid of the compact star and the power-law index, respectively. In order to initiate magnetically driven outflows, an azimuthal shearing motion is assumed around the equatorial surface, generating the azimuthal component of the magnetic field (Mikic \& Linker 1994). It is physically modeling the suppling process of the helical component of the magnetic field and magnetic energy from the compact star interior into the magnetosphere of the star.

\section{Results}

Figure 1 depicts the time evolution of the expanding outflow for the case where $\alpha=3.5$ and $\beta_{0}=3.2$. Here $\beta_{0}$ is the plasma beta on the equatorial surface. The density and the magnetic field line projected on the meridional plane are demonstrated at the time (a) $t=0$, (b) $300 \tau$ and (c) $700 \tau$ respectively. The normalization unit in time is the light crossing time $\tau \equiv R_{*} / c$ where $R_{*}$ and $c$ are central star radius and light speed, respectively. Note that the density is normalized by its initial value and filled by the gray contour in a logarithmic scale.

The magnetic pressure resulted from the azimuthal field component, which is generated by the shearing motion around the stellar equatorial surface, initiates and accelerates the quasi-spherical outflow. The driven outflow is strongly magnetized, in which the magnetic pressure mainly supported by newly generated toroidal field dominates the gas pressure, and carries dense plasma away from the compact object.

The magnetically driven outflow excites a strong forward shock as another outflow component, shock driven outflow. The forward shock expands supersonically into the surrounding medium and is accelerated to sub-relativistic velocity, $\sim 0.3 c$, in the case where $\alpha=3.5$ and $\beta_{0}=3.2$. 
We find that there exists a tangential discontinuity behind the propagating forward shock. The magnetic pressure provided by the toroidal magnetic field becomes predominant behind the discontinuity. In contrast, the gas pressure dominates the magnetic pressure between the discontinuity and the shock surface at all the evolutionary stage except a transit region near the discontinuity.

The physical properties of the magnetically driven outflow are examined with changing $\alpha$-parameter that controls the density profile. The magnetically driven outflow velocity $v_{\text {mag }}$ would be characterized by the strength of the initial dipole field which is the seed of the predominant toroidal field behind the tangential discontinuity.

Figure $2 \mathrm{a}$ shows the magnetically driven outflow velocity $v_{\text {mag }}$ in the relation with initial Alfvén velocity $v_{\mathrm{A}, 0}=B_{0} / \sqrt{4 \pi \rho_{0}}$ at the equatorial surface of the central star which is the representative of the seed field strength in the cases $\alpha=3.50,4.50,5.50$, 6.50 , and 7.00 respectively. Here $B_{0}$ and $\rho_{0}$ are the magnetic field strength and density on the compact star surface. The initial Alfvén velocity is measured at the equatorial surface of the star, and varies from $3.7 \times 10^{-3} c$ to $0.15 c$. The logarithmic fitting of the numerical data provides a simple scaling relation $v_{\mathrm{mag}} \propto v_{\mathrm{A}, 0}{ }^{1 / 2}$.

We focus on the dynamic balance of the system to draw a physical picture for accounting the scaling relation. Our numerical models suggest that at the final steady state, the strength of the toroidal magnetic field behind the tangential discontinuity does not significantly change in time. Hence the radial advection of the azimuthal magnetic field should counterbalance with the generation of the azimuthal field by the shearing motion near the stellar surface. Then we can give an induction equation in the steady form

$$
\begin{aligned}
\frac{\partial B_{\phi}}{\partial t} & =[\nabla \times(\mathbf{v} \times \mathbf{B})]_{\phi} \\
& \sim \frac{B_{0} \Delta v_{\phi}}{L}-\frac{B_{\phi, \text { near }} v_{r, \text { near }}}{R_{*}} \sim 0,
\end{aligned}
$$

where $B_{\phi, \text { near }}$ and $v_{r, \text { near }}$ are the toroidal magnetic field and radial fluid velocity in the region above the stellar surface where the toroidal magnetic flux is stored. $\Delta v_{\phi}$ represents the shearing velocity. The first term in the right hand side of eq (2.2) denotes the generation of the toroidal magnetic field by the shearing motion with the typical latitudinal width $L$. The second term shows the radial advection of the toroidal magnetic field. The toroidal magnetic field $B_{\phi}$,near near the stellar surface gives

$$
B_{\phi, \text { near }} \sim\left(\frac{R_{*}}{L}\right)\left(\frac{\Delta v_{\phi}}{v_{r, \text { near }}}\right) B_{0} .
$$

The magnetic pressure due to the azimuthal component of the magnetic field near the stellar surface should drive the outflow. Hence the kinetic energy of the outflow is powered by the magnetic energy mainly contributed from the toroidal field component,

$$
\frac{1}{2} \rho_{\mathrm{mag}} v_{\mathrm{mag}}{ }^{2}=\frac{B_{\phi, \text { near }}{ }^{2}}{8 \pi},
$$

where $\rho_{\text {mag }}$ and $v_{\text {mag }}$ are the typical density and velocity of the magnetically driven outflow, respectively. Since the typical velocity of the outflow is roughly provided by the radial advection velocity,

$$
v_{\text {mag }} \sim v_{r, \text { near }} .
$$

we can obtain, with combining equations (2.4) and (2.5) with (2.3),

$$
v_{\text {mag }} \sim v_{\mathrm{A}, 0}{ }^{1 / 2} \Delta v_{\phi}{ }^{1 / 2}\left(\frac{L}{R_{*}}\right)^{-1 / 2}\left(\frac{\rho_{\mathrm{mag}}}{\rho_{0}}\right)^{-1 / 4} .
$$



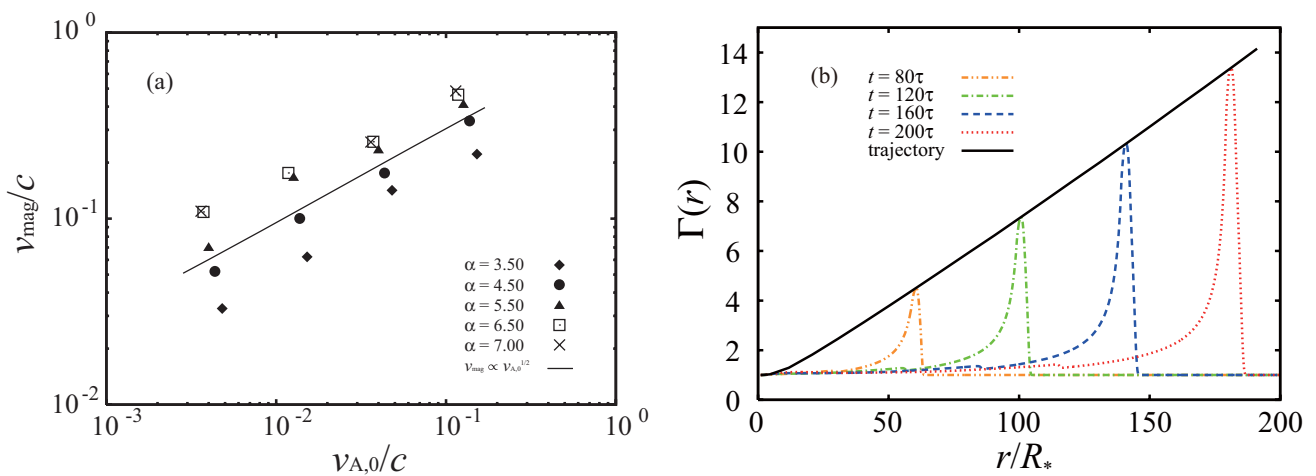

Figure 2. Panel (a): The relation between the Alfvén velocity at the equatorial surface and the velocity of the tangential discontinuity measured at $r=70 R_{*}$ when $\alpha=3.50,4.50,5.50,6.50$, and 7.00. There is a simple scaling relation between these two values, $v_{\mathrm{mag}} \propto v_{\mathrm{A}, 0}{ }^{1 / 2}$. Panel (b): The time evolution of the Lorentz factor of the expanding gas in the case with $\alpha=7.25$ and $\beta_{0}=3.2$. The dotted, dashed, dot-dashed, and dashed-two dotted curves indicate the cases where $t / \tau=80,120,160$, and 200 respectively. A solid line represents the time trajectory of the Lorentz factor of the fluid velocity at the shock surface measured in the laboratory frame.

Note that the parameters $v_{\mathrm{A}, 0}, \Delta v_{\phi}, L$ and $R_{*}$ are the parameters initially given. This indicates that the velocity of the magnetically driven outflow has a scaling relation $v_{\text {mag }} \propto$ $v_{A}^{1 / 2}$. We can exhibit the thick line in Figure $2 \mathrm{a}$ when the typical parameters which describes our numerical model are adopted. It is noted again that the two balancing equations, which are reduced from the induction and energy equations, account for this scaling relation.

When the initial density profile declines steeply with radius, the outflow-driven shock is accelerated to relativistic velocity ahead of the magnetically driven outflow. Figure $2 \mathrm{~b}$ illustrates the time evolution of the radial velocity profile in the case where $\alpha=7.25$ and $\beta_{0}=3.2$. It is found from this figure that the evolution of the relativistic shock has self-similar property. The fitting of numerical data provides a scaling relation between the Lorentz factor $\Gamma_{\mathrm{sh}}$ and the equatorial radius $r_{\mathrm{sh}}$ at the shock surface in the laboratory frame

$$
\Gamma_{\mathrm{sh}} \propto r_{\mathrm{sh}} .
$$

The Lorentz factor of the plasma velocity at the shock surface increases linearly with the equatorial radius. The self-similar property of the relativistic shock can be also reproduced by one-dimensional hydrodynamic model (Matsumoto et al. 2011). This thing suggests that the pure hydrodynamic process is responsible for the self-similar acceleration of the relativistic shock we found in the 2D MHD model.

\section{References}

Beskin, V. S. \& Nokhrina, E. E. 2006, MNRAS, 367, 375

Hayashi, M. R., Shibata, K., \& Matsumoto, R. 1996, ApJ (Letters), 468, L37

Komissarov, S. S. \& Lyubarsky, Y. E. 2004, MNRAS, 349, 779

Komissarov, S. S. 2006, MNRAS, 367, 19

Mikic, Z. \& Linker, J. A. 1994, ApJ, 430, 898

Spruit, H. C. 2010, Lecture Notes in Physics, Berlin Springer Verlag, 794, 233

Takahashi, H. R., Asano, E., \& Matsumoto, R. 2009, MNRAS, 394, 547

Thompson, C. \& Duncan, R. C. 1995, MNRAS, 275, 255 